\title{
Publisher Correction: Mucosal vaccines - fortifying the frontiers
}

\author{
Ed C. Lavelle (D) and Ross W. Ward (D)
}

Correction to: Nature Reviews Immunology https://doi.org/10.1038/s41577-021-00583-2, published online 26 July 2021.

In Figure 2 describing the 'Licensed mucosal vaccines' the green box detailing the rotavirus vaccines was incorrectly labelled as 'Poliovirus' in the originally published version. Further, in the peach "Vibrio cholerae" box, the composition for Vaxchora inadvertently duplicated text from the box above and has been amended to read "live attenuated 01 serogroup (Inaba) - ctxA attenuation." This has all now been corrected and we apologize for the errors.

https://doi.org/10.1038/s41577-021-00599-8 I Published online 3 August 2021

๑) Springer Nature Limited 2021, corrected publication 2022 